\title{
Increasing the imaging capabilities of the VLTI using integrated optics
}

Pierre Y. Kern, Fabien Malbet, Jean-Philippe Berger, Pierre Haguenauer, Isabelle Schanen-Duport, et al.

Pierre Y. Kern, Fabien Malbet, Jean-Philippe Berger, Pierre Haguenauer, Isabelle Schanen-Duport, Pierre Labeye, Karine Perraut-Rousselet, Brahim Arezki, Alain Delboulbe, Laurent Jocou, Christian Perrier-Bellet, Wesley A. Traub, Rafael Millan-Gabet, John D. Monnier, Ettore Pedretti, Andreas Glindemann, Pierre Kervella, Emmanuel di Folco, Philippe B. Gitton, "Increasing the imaging capabilities of the VLTI using integrated optics," Proc. SPIE 4838, Interferometry for Optical Astronomy II, (21 February 2003); doi: 10.1117/12.459074

Event: Astronomical Telescopes and Instrumentation, 2002, Waikoloa, Hawai'i, United States 


\title{
Increasing the imaging capabilities of the VLTI using integrated optics
}

\author{
Pierre $\operatorname{Kern}^{a}$, Fabien Malbet ${ }^{a}$, Jean-Philippe Berger ${ }^{a}$, Pierre Haguenauer ${ }^{b}$, \\ Isabelle Schanen ${ }^{b}$, Pierre Labeye ${ }^{c}$, Karine Rousselet-Perraut ${ }^{a}$, \\ Brahim Arezki $^{a}$, Alain Delboulbe ${ }^{a}$, Laurent Jocou ${ }^{a}$, Christian Perrier ${ }^{a}$, \\ IOTA collaboration: W. Traub, R. Millan-Gabet, J. Monnier, E. Pedretti \\ ESO collaboration: A. Glindemann, P. Kervella, E. Di Folco, P. Gitton \\ ${ }^{a}$ Laboratoire d'AstrOphysique de Grenoble, Grenoble, France \\ ${ }^{b}$ IMEP, INPG, Grenoble, France \\ ${ }^{c}$ LETI-CEA, Grenoble, France
}

\begin{abstract}
Several scientific topics linked to the observation of extended structures around astrophysical sources (dust torus around AGN, disks around young stars, envelopes around AGBs) require imaging capability with milli-arcsecond spatial resolution. The current VLTI instruments, AMBER and MIDI, will provide in the coming months the required high angular resolution, yet without actual imaging. As a rule of thumb, the image quality accessible with an optical interferometer is directly related to the number of telescopes used simultaneously: the more the apertures, the better and the faster the reconstruction of the image. We propose an instrumental concept to achieve interferometric combination of $\mathrm{N}$ telescopes $(4 \leq N \leq 8)$ thanks to planar optics technology: $4 \times 8$-m telescopes in the short term and/or $8 \times 1.8-\mathrm{m}$ telescopes in the long term. The foreseen image reconstruction quality in the visible and/or in the near infrared will be equivalent to the one achieved with millimeter radio interferometers. Achievable spatial resolution will be better than the one foreseen with ALMA.

This instrument would be able to acquire routinely 1 mas resolution images. A 13 to 20 magnitude sensitivity in spectral ranges from 0.6 to $2.5 \mu \mathrm{m}$ is expected depending on the choice of the phase referencing guide source. High dynamic range, even on faint objects, is achievable thanks to the high accuracy provided by integrated optics for visibility amplitude and phase measurements. Based on recent validations of integrated optics presented here an imaging instrument concept can be proposed. The results obtained using the VLTI facilities give a demonstration of the potential of the proposed technique.
\end{abstract}

Keywords: Interferometry, integrated optics, guided optics, closure phase

\section{INTRODUCTION}

While large interferometers like the VLTI or the Keck become operational, and other instruments with smaller apertures produce routinely scientific results, it can be considered that optical interferometry is becoming a mature technique both on the technological and scientific sides. The astrophysics targets that have been investigated so far range from stellar physics to the calibration of the extragalactic scale: stellar parameters $(R$, $\left.T_{\text {eff }}, \ldots\right)$, shells of late-type stars, Be/P Cyg shells, binary star orbits, stellar atmosphere, novae, cepheids, young stellar objects,... with only 6 to 7 operational interferometers. The reduced apertures of operational interferometers limit the achievable sensitivity reducing the number of possible targets. The large interferometers will undoubtedly enlarge the scientific field to fainter sources, even to extragalactic targets.

In this framework, the VLTI is a unique facility with its main array composed of $4 \times 8$-m fixed telescopes with a maximum baseline of $130 \mathrm{~m}$, its subarray of $3 \times 1.8-\mathrm{m}$ relocable telescopes with a maximum baseline of $200 \mathrm{~m}$ and 3 delay lines. The subarray has been designed ${ }^{1}$ to be extended to 8 auxiliary telescopes (see also ESO

Further author information: (Send correspondence to Pierre Kern)

E-mail: Pierre.Kern@obs.ujf-grenoble.fr, Telephone: +33476 635841

Address: LAOG, BP 53, 38041 Grenoble Cedex 9, France 

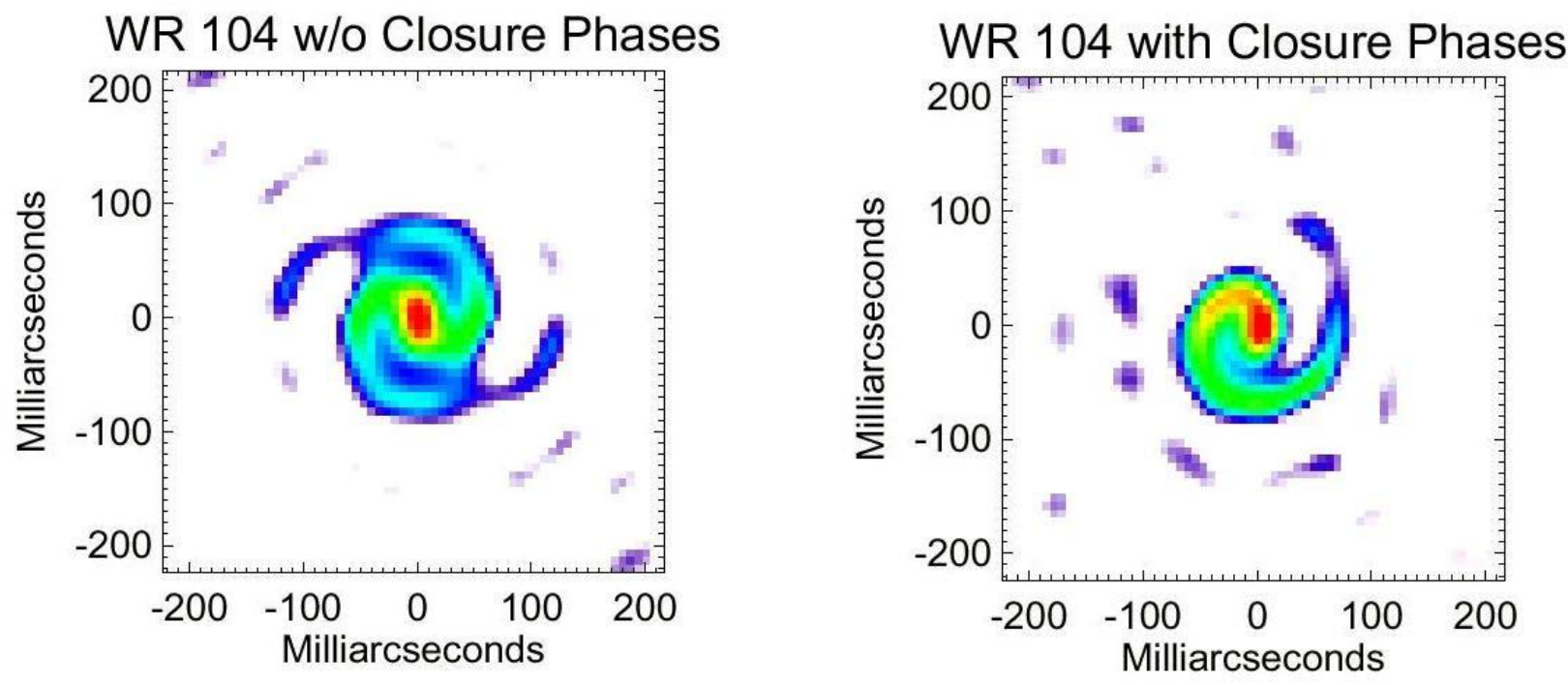

Figure 1. The Wolf-Rayet star WR104 observed by aperture masking (technique that simulates an array of telescopes with a single telescope) on one of the Keck telescope. Left panel: reconstructed image without the phases; right panel: reconstructed image with the phases (from Monnier $1999^{4}$ ).

contribution in this proceedings). The first VLTI instrument generation gives access to the combination of 2 telescopes in the $8-20 \mu \mathrm{m}$ range (MIDI ) or 3 telescopes in the $1-2.5 \mu \mathrm{m}$ range with a spectral resolution up to 10000 (AMBER). The observables will mainly be visibility amplitudes or relative phases, as well as one closure phase for AMBER. However a more efficient imaging capacity is mandatory for complex astronomical fields, like the accretion-ejection region in disks of young stellar objects, compact stellar clusters or the inner part of active galactic nuclei.

Some of the important astrophysical targets like the central engine of galaxies, the close environment of young stellar objects, the formation of extrasolar planets or the shells around late-type stars, constrains the requirements for a second generation VLTI instrument:

- important imaging capability (in terms of number of pixels in the image): $n \geq 100$.

- high sensitivity: $K \geq 13$

- extended spectral coverage down to $\mathrm{H}_{\alpha}(0.65 \mu m)$

- larger field of view: FoV $\geq 1 "$

In this context, we propose to build a multi-beam combining instrument for imaging at low spectral resolution $(\sim 100)$, i.e. an instrument that combines up to 8 beams, working from $0.8 \mu m$ to $2.5 \mu m$ with possible extensions in the $[0.6-5 \mu \mathrm{m}]$ range. This will provide an excellent imaging capability thanks to multi beam operation, high sensitivity while using an array of very large telescopes and high efficiency thanks to a snapshot observing mode.

Integrated optics offers a unique solution to meet such requirements. It is able to provide several designs in extremely compact and easy to implement setup. Main elementary blocks have already been succesfully tested in laboratory and on the sky.

\section{IMAGING, AN IMPORTANT ISSUE FOR INTERFEROMETRY}

When dealing with complex structures, one usually requires non-a-priori information on the target: this is achieved by imaging. Actually interferometers provide measurements of complex visibilities or related quantities. 

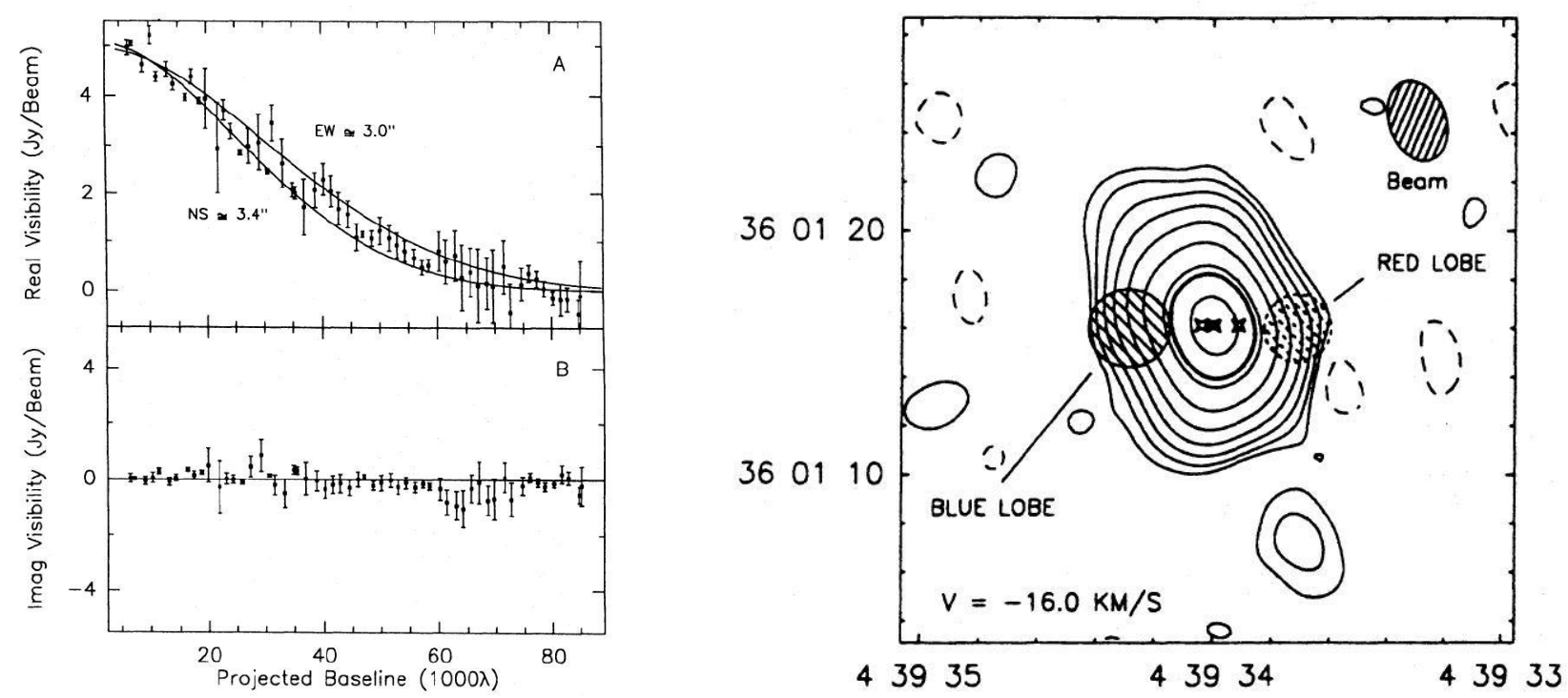

Figure 2. Image of the protoplanetary nebula CRL 618 (right side) reconstructed from the measured visibilities (left side) using 6 configurations of 3 antennas at IRAM. ${ }^{3}$

However for imaging, the knowledge of both the amplitude and the phase of the complex visibilities is required. In the optical domain, there are usually no problems with the amplitudes, but because of the atmospheric perturbation we are not able to calibrate the phases which are jittering. To demonstrate the importance of the phase on the reconstructed image, Figure 1 presents two images obtained respectively without and with the phase information. Without the phase information, one sees that we can make important errors.

The step of imaging is achieved with radio interferometers for any observations. Moreover even with a limited number of telescopes, it is possible to achieve imaging as observed for the proto-planetary nebula CRL $618^{3}$ at IRAM with 6 configurations of only 3 antennas (see Figure 2).

Currently visibility phases are obtained with optical interferometers either by external or differential phase referencing, or more usually using closure phases with at least 3 telescopes.

The closure phase is the sum of the phases obtained over the baselines of a triplet of telescopes. All observational phase errors like the atmospheric jitters are then canceled. The higher the number of telescopes, and therefore of independent closure phases, the better the quality of image reconstruction. The efficiency of an array is given by the number of spatial frequencies that can be retrieved simultaneously: for $N$ telescopes in a non-redundant array, one can measure $N(N-1) / 2$ spatial frequencies. Using closure phases, one is able to retrieve $100 \times(1-2 / N)$ percent of the phase information. To reconstruct a full image, one also needs to have additional constraints like image positivity or limited support of the object.

Figure 3 shows the number of theoretically accessible phase points with various number of telescopes and also the number of independent closure phases. The curve on this figure shows the corresponding percentage of phase information that can be retrieved.

Figure 3 illustrates that $N=4$ and $N=8$ allow to provide respectively $50 \%$ and $75 \%$ of the phase information. To increase significantly the percentage of phase information, one would be obliged to use much more telescopes because of the asymptotic behaviour of the phase information. The third column of the table (Figure 3 ) gives the gain in efficiency (increase of the number of accessible spatial frequencies) compared to a 3-telescopes combination. It ranges respectively from a factor 2 for 4 beams to almost 10 for 8 ones.

Up to know only three interferometers are able to achieve imaging with $N \geq 3$ : IOTA with three apertures, COAST with 5 apertures and NPOI with 6 apertures. However only a small number of results have been 


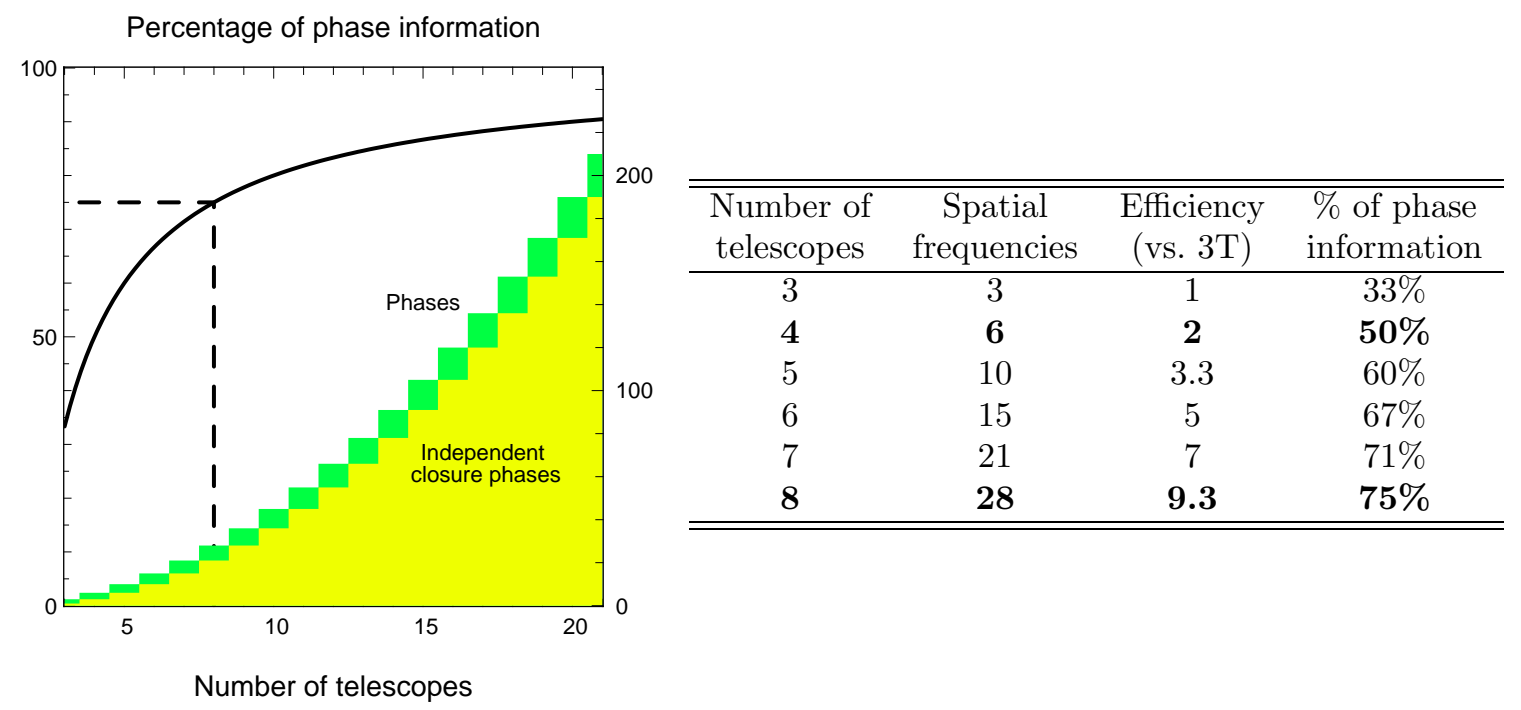

Figure 3. Left: histogram of the number of accessible phases (black) and independent closure phases (gray). The curve gives the percentage of phase information retrieved versus the number of telescopes. Table at right: snapshot efficiency and percentage of phase information retrieved by closure phases for array ranging from 3 to 8 telescopes. The third column gives the improvement compared to a 3 telescope array.

obtained $^{5,6}$ so far, because of the complexity of the related instrumentation. Indeed, the stability of the beam combiner is one of the critical issue while the number of its optical surfaces increases as $\sim N^{2}$. That is why the number of telescopes used in array remains small and why COAST has developed a new type of beam combiner with small glued optics. ${ }^{7}$ We believe that the technology of integrated optics provides new solutions which will allow to combine a higher number of beams.

\section{INTEGRATED OPTICS AND ASTRONOMICAL INTERFEROMETRY}

\subsection{Available technologies}

Integrated optics (IO) has been proposed in 1996 as a solution for beam combiner instruments in interferome$\operatorname{try}{ }^{8,10}$ and at this time encouraging results have been obtained in laboratory and on the sky using 2 or three telescopes. A general description of IO as used in interferometry is given by Jean Philippe Berger in this volume.

Developed for telecommunication and sensor applications, this technology is presently mature and commercially available for wavelengths ranging from $0.8 \mu \mathrm{m}$ to $1.8 \mu \mathrm{m}$. The provided stability and high integration capability of optical functions allow to envision combining the light from 4 to 8 telescopes on a single chip, of typically a few squared centimeters large. This technology can be used therefore as a basis for a VLTI second generation instrument.

Integrated Optics technology allows to provide beam propagation within an optical chip using optical waveguide or optical guiding structures. Several technologies are used to manufacture the components thanks to the classical microphotolithography processes used in micro-electronics. Ion exchange, silica etching (respectively IET and SET hereafter) and $\mathrm{LiNbO3}$ structures are the most used. ${ }^{8,9}$ When using components including single mode waveguides, spatial filtering of the light is achieved leading to excellent calibration of the incoming flux on each telescope with respect to the atmospheric turbulence.

The microphotolithography process gives access to multiple optical functions providing usual optical operation on the propagated beams as division, combination, focusing in some conditions, but also diffraction or phase modulation, etc. A whole set of functions is available to design beam combiners for $\mathrm{N}$ telescopes ${ }^{9,10}$ using either multiaxial or coaxial principle. All the difficulty of the combination of multiple beams ${ }^{11}$ is reported in the design stage of the lithography mask, to ensure the arrangement of the waveguide tracks and manage properly the 


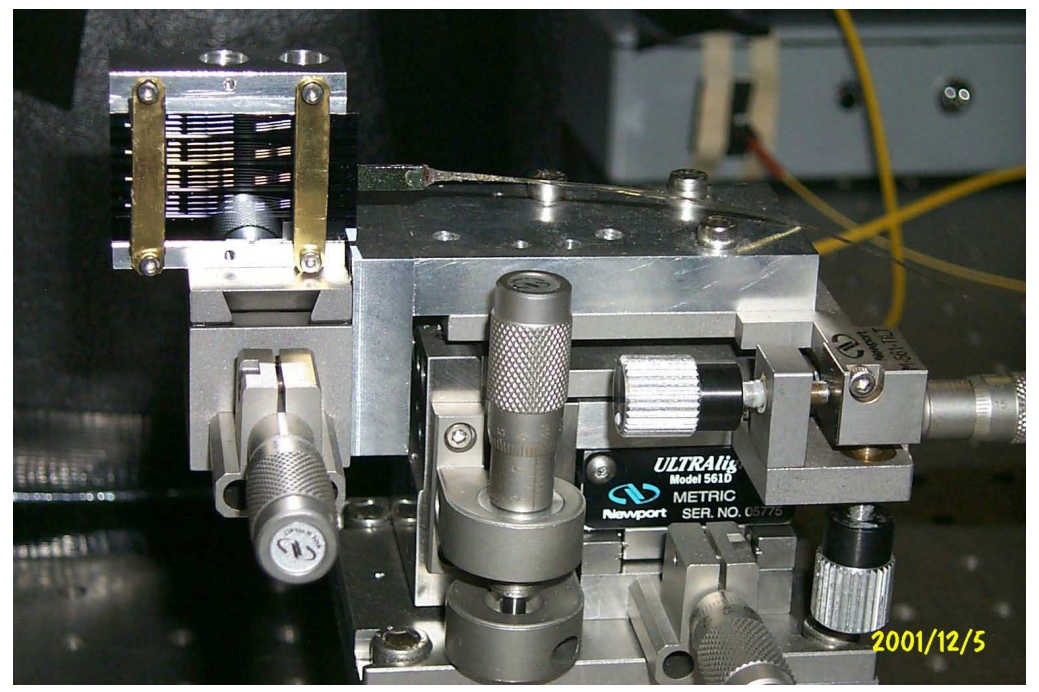

Figure 4. Three telescope beam combiner made with integrated optics using SET used on IOTA. The light from the three telescopes is injected in the component using three polarization maintaining single mode fibers (fiber optics on the right side); the light is combined thanks to asymetrical directional couplers, providing for each baseline two interferometric outputs in phase opposition. Component outputs (left side of the picture) are directly imaged on an IR camera.

critical parameters of the light: polarization, dispersion, and phase. Current process of available technology designed for telecommunication or micro-sensors, i.e. $0.8,1.3$ and 1.55 microns, is directly applicable to the $I, J$ and $H$ near infrared atmospheric band. Manufacturing components for other wavelength bands requires specific developments. An extension to $0.6 \mu \mathrm{m}$ and $2.2 \mu \mathrm{m}$ requires an adaptation of existing processes. Validation of both SET and IET 2 telescopes components used in the $K$ band have been performed thanks to laboratory and on the sky experiments, ${ }^{18}$ with excellent performances as reported below in the next section. Adaptation for shorter wavelengths is in progress but still needs to be validated. To be able to manufacture operational waveguides for the thermal infrared, $\mathrm{R} \& \mathrm{D}$ on suitable materials is still needed. Dedicated funding are allocated to this development by space agencies (CNES, ESA and NASA) to obtain such single mode waveguides in the scope of the DARWIN and TPF programs.

\subsection{Laboratory validations for astronomical interferometry}

Characterization of various beam combiners were performed ${ }^{13,14,18}$ in laboratory. Excellent performances of integrated optics components were pointed out according to the tested designs:

- high contrast (97\% in the $H$ band and $93 \%$ in the $K$ band when selecting one of the polarization state, and $90 \%$ without polarization control in the $H$ band),

- high optical throughput greater than $60 \%$ in the $H$ band,

- non-detectable chromatic dispersion,

- good behaviour in polarization because of the polarization maintaining properties of the waveguides

- extremely good thermal stability: a relative change of $1.6 \times 10^{-6}$ of the optical path for 1 degree of temperature change. ${ }^{11}$

Some of these results can be affected by the fiber optics associated to the integrated optics components for light injection, mainly due to their polarization behavior. Developments are in progress on beam combiners to achieve the best performances on the same optimized designs. In depth simulations, design and characterization were also performed in the framework of the Darwin project. ${ }^{15}$ Provided simulations shows the capability of 

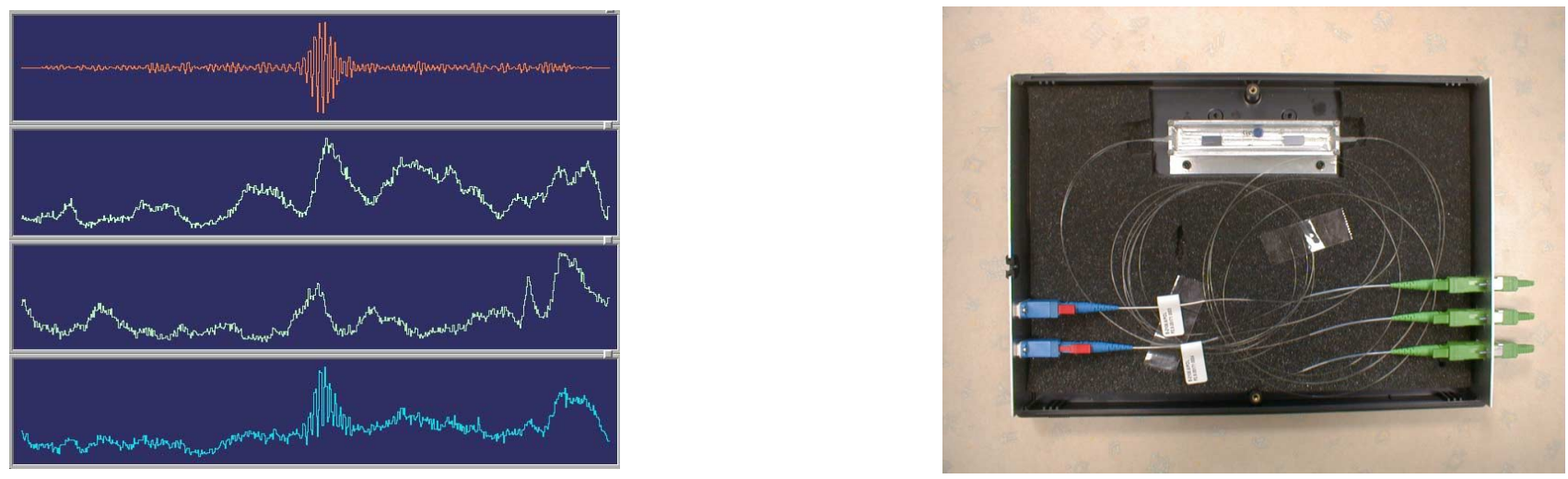

Figure 5. left: Fringes obtained on Altair using a $57 \mathrm{~m}$ baseline of two $8 \mathrm{~m}$ telescopes of the VLTI array (UT1-UT2). The above fringe pattern is corrected from photometric fluctuations at the entrance of each feeding fiber optics (middle interferograms) as the bottom pattern is the uncorrected interferogram. right: IO component installed on the VINCI VLTI instrument to provide it with $H$ band capabilities. The two left connectors are used for light injection from the telescope, and the three right connectors are linked to the existing VINCI camera interface. The beam combiner itself is the small glass sheet on the top of the picture.

guided optics to achieve spatial filtering according to the required specifications for nulling interferometry. ${ }^{16}$ Two set of 2 telescope beam combiners were provided (IET and SET components), with an optimized design for their integration on a nulling interferometry bench.

\subsection{On site validations}

\subsubsection{IOTA tests}

Both 2 telescope and 3 telescope beam combiners were used on IOTA. Two components (SET and IET) were used in the IOTA 2 telescope configuration and fringes were recorded on 14 stars during 4 observing nights. ${ }^{17}$ Switching time between the two components took less than 2 hours, mainly due to polarization adjustments on injecting fiber optics. Instrumental contrasts better than $60 \%$ were achieved. Implementing polarization control in the setup can provide even better contrasts. The throughput was limited by an unadapted injection optics, leading to a faintest observed star of $H \sim 2$; rough tests using the SET component allow to obtain fringes in the $K$ band.

The current IOTA 3 telescope beam combiner instrument uses an integrated optics SET component. Installed in 2001 on IOTA, it was used as a debug instrument during the extension to the third telescope. Closure phases are obtained now, and the instrument is proposed to the IOTA users. First obtained results are reported by Jean Philippe Berger in these proceedings. ${ }^{20}$

\subsubsection{VLTI -VINCI instrument improvement}

During a 7 nights run on VLTI (July 17-23, 2002) an integrated optics interferometric beam combiner was installed on the VLTI commissioning instrument VINCI. This combiner, manufactured by GeeO (Grenoble France), provides VINCI with $\mathrm{H}$ band capability in addition to its usual fluoride glass fiber beam combiner dedicated to the $\mathrm{K}$ band (MONA). In the tested configuration the beams from the two $35 \mathrm{~cm}$ siderostats of the VLTI, with a 66 mbaseline, were combined.

For this first run, the installation of the beam combiner was achieved without any major modification of the optical and software interfaces of VINCI. The hardware installation was truly plug-and-play, first unplugging the MONA fibers and then plugging the fibers of this new component, taking less than five minutes. After this easy installation during the first night, fringes were obtained in VLTI laboratory with an internal source and then on sky measurements were performed during all the nights of the run as for usual observing nights of the VINCI instrument. 
We obtained fringes on the sky in the atmospheric $\mathrm{H}$ band $(1.4-1.8 \mu \mathrm{m})$ with a visibility accuracy of $1 \%$. Interferometric transfer functions of the VLTI / IONIC were consistantly measured to be above $80 \%$. The data were reduced online with the VINCI pipeline. The component contrast as previously measured in the laboratory was $90 \%$ for unpolarized light.

The faintest observed star is $\kappa 2 \mathrm{Scl}$ (HD 720) with a magnitude $H \sim 3.1$. It is currently difficult to determine the final limiting magnitude due to the mismatch between fiber optics numerical aperture and feeding optics. Optimization of the overall transmission would require to change the injection optics of VINCI to be adapted to the numerical aperture of the new injection fibers (0.12 compared to 0.23 for MONA fluoride fiber optics manufactured for $K$ band operation).

Thanks to this promising results, the integrated optics beam combiner was used to obtain first fringes on the UT1-UT2 (57m) VLTI baseline (see Figure 5).

The promising results further confirm the flexibility and performances of the IO technology in the astronomical interferometry field.

\subsection{Instrumental considerations}

Regarding to an imaging instrument, a great advantage of IO components as beam combiners is their stability able to provide an accurate measurement of the closure phase as required to obtain high dynamic ranges in the reconstructed image.

For spectral dispersion it can be noticed that the output beams of planar guides can be placed at the location of the entrance slit of a spectrograph avoiding complex cylindric optical setup.

As for radio-interferometers, the field of view is limited by the diffraction lobe of the telescopes, while only the Airy pattern feeds the single mode waveguide (60 mas for the UTs and 250 mas for the ATs), and comparable mosacing techniques can be used to increase the final field of view.

In summary we can state that integrated optics is a key technology for interferometric imaging since it is able to provide:

- easy combination of high number of beams and ability to reconfigure the combination scheme (e.g. for non-redundant array)

- good performance, both in fringe contrast and optical throughput

- reliable and stable (everything integrated onto a single piece of material)

- based on products from the telecommunication industry

- works already at $\lambda=0.8,1.3,1.5$ and $2.2 \mu \mathrm{m}$ and potentially at $\lambda \leq 0.8 \mu \mathrm{m}$ and $\lambda \geq 2.5 \mu \mathrm{m}$

\section{PROPOSITION FOR A VLTI IMAGING INSTRUMENT}

Among the other existing interferometers, the VLTI will be the first one to be able to achieve imaging on faint stars thanks to its four 8-m class telescopes, compared to the Keck interferometer with two 10-m telescopes and four 1.8-m outriggers, CHARA with six 1-m telescopes and OHANA that will combine the large telescopes at the Mauna Kea summit.

Following our experience in interferometry and the VLTI already existing infrastructure, we propose a strategy in two stages:

- in the short term, a simplified imaging setup demonstrator for the main array ( $4 \times \mathrm{UTs})$ working from 0.8 to $2.5 \mu \mathrm{m}$ (see for example Figure 6), using the VINCI instrumental environment. The required infrastructure is limited: the optomechanical basis is available and requires only limited adaptations, 4 adaptive optics already funded, 1 additional delay line (if a fixed one is not already there), 1 additional fringe sensor. 

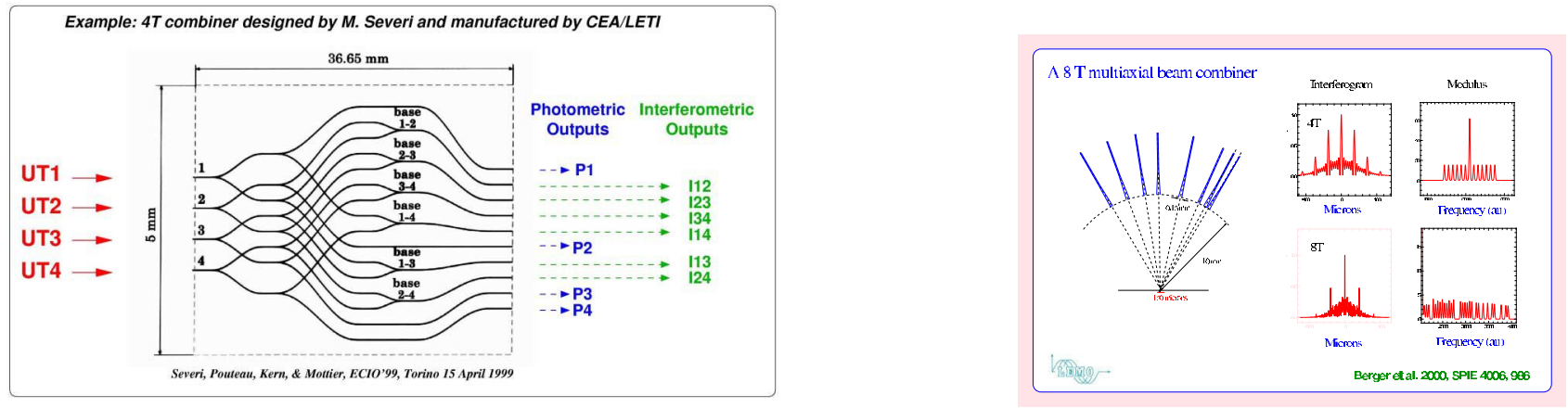

Figure 6. Existing elements for a possible configuration for a second generation instrument for the VLTI. Left: a 4-way component to combine the light of the 4 UTs. ${ }^{19}$ Right: proposed concept for an 8 telescope beam combiner. ${ }^{11}$

- in the long term, an imaging instrument for the extended array with possibly 8 telescopes (additional ATs or combination of ATs with some UTs) working from 0.6 to $5 \mu \mathrm{m}$. The required infrastructure is bigger: additional telescopes with a total of 6 to 8 ATs, a total of 8 delay lines, low order adaptive optics for the ATs if extension to the visible domain (with about 50 actuators), and a cophasing instrument, possibly using IO technology. Full system studies are required to achieve the best instrumental concept and related developments as the fringe tracking or dispersion requirements.

All proposed development would benefit from the VINCI/AMBER heritage, for instance control system and injection optics.

\section{ACKNOWLEDGMENTS}

Authors wish to thanks all members of the IONIC team at LETI, IMEP and LAOG, and GeeO. All results presented here where obtained with components manufactured with IMEP, LETI and GeeO facilities. Part of this work has been funded by CNES and CNRS / INSU. Development related to nulling breadboard is funded by ESA in the framework of the DARWIN mission.

\section{REFERENCES}

1. A. Glindemann et al.: The ESO Messenger 98, 2, 1999.

2. P.G. Tuthill, J.D. Monnier, W.C. Danchi, E.H. Wishnow, C.A. Haniff: PASP 112, 555, 2000.

3. R. Neri, S. Garcia-Burillo, M. Guelin, J. Cernicharo, S. Guilloteau, R. Lucas: A\&A 262, 544, 1992.

4. J.D. Monnier: "An introduction to Closure Phases". In Principles of Long Baseline Stellar Interferometry, Michelson Summer School 1999, ed. by P.R. Lawson, pp. 203-229, 1999.

5. J. S. Young, J. E. Baldwin, R. C. Boysen, C. A. Haniff, P. R. Lawson, C. D. Mackay, D. Pearson, J. Rogers, D. St.-Jacques, P. J. Warner, D. M. A. Wilson, R. W. Wilson: MNRAS 315, 635, 2000.

6. C. A. Hummel, D. Mozurkewich, J. T. Armstrong, A. R. Hajian, N. M. Elias, and D. J. Hutter: AJ 116, 2536, 1998.

7. C. A.Haniff, J. E. Baldwin, R. C. Boysen A. V. George, D. F. Buscher, C. D. Mackay, D. Pearson, J. Rogers, P. J. Warner, D. M. Wilson, and J. S.Young, "COAST: the current status", In: Interferometry in optical astronomy, SPIE'2000, Munich, Germany, ed. by P. Léna, A. Quirrenbach, vol. 4006, pp 627-633, 2000.

8. F. Malbet, P. Kern, I. Schanen-Duport, J.-P. Berger, K. Rousselet-Perraut, P. Benech: "Integrated optics for astronomical interferometry. I- Concept and astronomical applications", $A \& A S$ 138, 131, 1999.

9. P. Kern, J.-P. Berger, P. Haguenauer, F. Malbet, K. Rousselet-Perraut: "Planar integrated optics contribution to instrumentation for interferometry". In: Interferometry in optical astronomy, SPIE'2000, Munich, Germany, ed. by P. Léna, A. Quirrenbach, vol. 4006, pp 974, 2000. 
10. P. Kern, J.-P. Berger, P. Haguenauer, F. Malbet, K. Rousselet-Perraut, "Planar integrated optics and astronomical interferometry". C.R. Acad. Sci. Paris 2, Ser. IV, 111, pp. 111-124, 2001.

11. J.-P. Berger, P. Benech, I. Schanen, G. Maury, F. Malbet, F. Reynaud, "Combining up to eight telescope beams in a single chip". In: Interferometry in optical astronomy, SPIE'2000, Munich, Germany, ed. by P. Léna, A. Quirrenbach, vol. 4006, pp 986, 2000.

12. J.-P. Berger, I. Schanen-Duport, S. El-Sabban, P. Benech, M. Severi, P. Pouteau, K. Rousselet-Perraut, P. Haguenauer, Y. Duchene, F. Malbet, P. Kern, "Integrated optics beam combiners for astronomical aperture synthesis". In: Working on the fringe, Dana Point, May 1999, ed. by S. Unwin, pp $264,1999$.

13. J.-P. Berger, K. Rousselet-Perraut, P. Kern, F. Malbet, I. Schanen-Duport, F. Reynaud, P. Haguenauer, P. Benech, "Integrated optics for astronomical interferometry, II- First laboratory white-light interferograms", $A \xi A S, \mathbf{1 3 9}, 173,1999$.

14. P. Haguenauer, J.-P. Berger, K. Rousselet-Perraut, P. Kern, F. Malbet, I. Schanen-Duport, P. Benech, "Integrated optics for astronomical interferometry. III- Optical validation of a planar optics two-telescope beam combiner", Appl. Opt. 39 (13), 2130, 2000.

15. P. Haguenauer, M. Barillot, P. Kern, I. Schanen-Duport, V. Collomb, P. Labeye, A. Poupinet, V. Weber, Z. Sodnik, P. Kervella, "Nulling interferometric breadboard using integrated optics beam combiners, preparation to the IRSI/DARWIN mission", In these proceedings: Interferometry for optical astronomy II, SPIE'2002, Kona, Hawaii, ed. by W. Traub, vol. 4838, 2002

16. P. Leproux, V. Weber, I. Schanen-Duport, P. Haguenauer, V. Doya, F. Reynaud, P.Benech, J.-E. Broquin, P. Kern, "Numerical simulations on spatial filtering efficiency with optical fibers and integrated optics components", In these proceedings: Interferometry for optical astronomy II, SPIE'2002, Kona, Hawaii, ed. by W. Traub, vol. 4838, 2002

17. J.-P. Berger, P. Haguenauer, P. Kern, K. Perraut, F. Malbet, I. Schanen, M. Severi, R. Millan-Gabet, W. Traub, "Integrated optics for astronomical interferometry. IV- First measurements of stars", $A \xi A$, 376, L31, 2001.

18. E. Laurent, K. Rousselet-Perraut,P. Benech, J.-P. Berger, S. Gluck, P. Haguenauer, P. Kern, F. Malbet, I. Schanen-Duport, "Integrated optics for astronomical interferometry - V. Extension to the K band", $A \mathscr{E} A$ - 1171, Volume 390, Number 3, 2002.

19. M. Severi, P. Pouteau, P. Mottier, P. Kern, "A waveguide interferometer for phase closure in astronomy". In: Proceedings of the ECIO'99 conference, Turin, Italy, pp 2791, 1999.

20. J.-P. Berger, P. Haguenauer, P. Kern, F. Malbet, K. Perraut-Rousselet, I. Schanen-Duport, M. Severi, R. Millan-Gabet, J. Monnier, E. Pedretti, W. Traub,"First results from the IONIC three beam combiner at IOTA", In these proceedings: Interferometry for optical astronomy II, SPIE'2002, Kona, Hawaii, ed. by W. Traub, vol. 4838, 2002 